\title{
CONTINUITY PROPERTIES OF MONOTONE NONLINEAR OPERATORS IN LOCALLY CONVEX SPACES
}

\author{
DIMITRIOS KRAVVARITIS
}

\begin{abstract}
Let $X$ be a real locally convex Hausdorff space, $X^{*}$ its dual space, and $T$ an operator from $X$ into $2^{X^{*}}$. The main results of this paper are: (i) if $T$ is $D$-maximal monotone and locally bounded at each point of $D(T)$, then $T$ is upper demicontinuous; (ii) if $X$ is a Fréchet space, $T$ is monotone, and $D(T)$ is open in $X$, then $T$ is upper demicontinuous if and only if $T$ is upper hemicontinuous, thus generalizing a result of [3].
\end{abstract}

Let $X$ be a real locally convex Hausdorff (topological vector) space, $X^{*}$ its dual space, and $2^{X^{*}}$ the space of subsets of $X^{*}$. We write $\left\langle u^{*}, u\right\rangle$ in place of $u^{*}(u)$ for $u \in X$ and $u^{*} \in X^{*}$. Let $T$ be an operator from $X$ into $2^{X^{*}}$. The effective domain of $T$ is the set

$$
D(T)=\{u \in X: T(u) \neq \varnothing\}
$$

and the graph of $T$ is the subset of $X \times X^{*}$ given by

$$
G(T)=\left\{\left(u, u^{*}\right): u^{*} \in T(u)\right\} .
$$

$T$ is called a monotone operator if $\left\langle u^{*}-v^{*}, u-v\right\rangle \geqslant 0$ for each pair of elements $\left(u, u^{*}\right)$ and $\left(v, v^{*}\right)$ of $G(T)$. T is called a $D$-maximal monotone operator [1] if, in addition, the following condition is satisfied: if $u \in D(T)$ and $u^{*} \in X^{*}$ such that $\left\langle u^{*}-v^{*}, u-v\right\rangle \geqslant 0$ for all $\left(v, v^{*}\right) \in G(T)$, then $u^{*} \in T(u)$. The operator $T$ is said to be locally bounded at $u_{0}$ if there exists a neighborhood $U$ of $u_{0}$ such that the set

$$
T(U)=\bigcup\{T(u): u \in U\}
$$

is an equicontinuous subset of $X^{*}$.

The domain $D(T)$ of $T$ is said to be quasi-dense [4] if for each $u \in D(T)$ there exists a dense subset $M_{u}$ of $X$ such that whenever $v \in M_{u}$, then $u+t v \in D(T)$ for sufficiently small $t>0$.

Let $X$ and $Y$ be topological spaces; an operator $T$ from $X$ into $2^{Y}$ is said to be upper semicontinuous if, for each $u_{0}$ in $X$ and each neighborhood $V$ of $T\left(u_{0}\right)$ in $Y$, there exists a neighborhood $U$ of $u_{0}$ such that $T(u) \subset V$ whenever $u \in U$.

If $X$ is a locally convex Hausdorff space and $X^{*}$ its dual, with $X^{*}$ given the weak* topology, then (i) an operator which is upper semicontinuous from $X$

Received by the editors July 5, 1977 and, in revised form, March 20, 1978.

AMS (MOS) subject classifications (1970). Primary 47H05; Secondary 46A05.

Key words and phrases. Monotone and $D$-maximal monotone operators, local boundedness, quasi-denseness, upper demicontinuity, upper hemicontinuity.

(1) American Mathematical Society 1978 
into $2^{X^{*}}$ is said to be upper demicontinuous and (ii) an operator which is upper semicontinuous from each segment $S \subset X$ into $2^{X^{*}}$ is said to be upper hemicontinuous.

We use the symbols " $\rightarrow$ " and " $\rightarrow$ " to denote strong and weak" convergence, respectively.

THEOREM 1. Let $X$ be a locally convex Hausdorff space and T a D-maximal monotone operator from $X$ into $2^{X^{*}}$ which is assumed locally bounded at each point of $D(T)$. Then $T$ is upper demicontinuous.

Proof. Suppose that $T$ is not upper demicontinuous. Then there exists a point $u_{0}$ in $D(T)$ and a weak* neighborhood $W$ of $T\left(u_{0}\right)$ such that for each neighborhood $U$ of $u_{0}$ there exist $u \in U$ and $u^{*} \in T(u)$ with $u^{*} \notin W$. Since $T$ is locally bounded at the point $u_{0}$, we conclude the existence of a neighborhood $V$ of $u_{0}$ such that the set $T(V)$ is an equicontinuous subset of $X^{*}$. Hence there exist a net $\left\{u_{\alpha}\right\}$ in $V$ with $u_{\alpha} \rightarrow u_{0}$ and $u_{\alpha}^{*} \in T\left(u_{\alpha}\right)$ such that $u_{\alpha}^{*} \notin W$ for all $\alpha$. Since the set $T(V)$ is relatively weak*-compact, there exists a subnet $\left\{u_{\beta}^{*}\right\}$ of $\left\{u_{\alpha}^{*}\right\}$ with $u_{\beta}^{*} \rightarrow u_{0}^{*} \notin T\left(u_{0}\right)$. By the monotonicity of $T$, we know that for every $\left(u, u^{*}\right) \in G(T)$ and every index $\beta$ we have $\left\langle u_{\beta}^{*}-u^{*}, u_{\beta}-u\right\rangle \geqslant 0$. But $u_{\beta} \rightarrow u_{0}$ and $u_{\beta}^{*} \rightarrow u_{0}^{*}$, so that the last inequality gives

$$
\left\langle u_{0}^{*}-u^{*}, u_{0}-u\right\rangle \geqslant 0
$$

for all $\left(u, u^{*}\right)$ in $G(T)$. The $D$-maximal monotonicity of $T$ implies that $u_{0}^{*} \in T\left(u_{0}\right)$, contrary to the fact that $u_{0}^{*} \notin T\left(u_{0}\right)$. Therefore, $T$ is an upper demicontinuous operator.

TheOREM 2. Let $X$ be a locally convex Hausdorff space and $T$ a monotone operator from $X$ into $2^{X^{*}}$ with a quasi-dense domain $D(T)$. Suppose that $T$ is upper hemicontinuous from $X$ into $2^{X^{*}}$ and for each $u \in D(T), T(u)$ is an equicontinuous, weak ${ }^{*}$-closed and convex subset of $X^{*}$. Then $T$ is $D$-maximal monotone.

Proof. Let $u_{0} \in D(T)$ and $u_{0}^{*} \in X^{*}$ such that $\left\langle u^{*}-u_{0}^{*}, u-u_{0}\right\rangle \geqslant 0$ for all $\left(u, u^{*}\right) \in G(T)$. It is sufficient to show that $u_{0}^{*} \in T\left(u_{0}\right)$. Suppose this is not the case. Consider $X^{*}$ endowed with the weak* topology. Then the dual of $X^{*}$ can be identified with $X$. Let $M_{u_{0}}$ be the dense subset of $X$ used in the definition of $D(T)$ as a quasi-dense set. Since $T\left(u_{0}\right)$ is an equicontinuous, weak*-closed and convex subset of $X^{*}$, there exists a $v \in M_{u_{0}}$ such that

$$
\left\langle w^{*}-u_{0}^{*}, v\right\rangle<0
$$

for all $w^{*} \in T\left(u_{0}\right)$. Furthermore, there exists a $t_{0}(v)>0$ such that $u_{t}=u_{0}+$ tv lies in $D(T)$ for $0<t<t_{0}(v)$. Let $\left\{t_{n}\right\}$ be a null sequence of real numbers such that $0<t_{n}<t_{0}(v)$ for all $n$. Then $u_{n}=u_{0}+t_{n} v \in D(T)$, and for any $u_{n}^{*} \in T\left(u_{n}\right)$ we have $t_{n}\left\langle u_{n}^{*}-u_{0}^{*}, v\right\rangle \geqslant 0$, i.e.,

$$
\left\langle u_{n}^{*}-u_{0}^{*}, v\right\rangle \geqslant 0
$$


for all $n$. By the upper hemicontinuity of $T$ the set $\cup_{n} T\left(u_{n}\right)$ is relatively weak ${ }^{*}$-compact, and so there exists a subnet $\left\{u_{\beta}^{*}\right\}$ of $\left\{u_{n}^{*}\right\}$ such that $u_{\beta}^{*} \rightarrow u_{1}^{*}$ $\in T\left(u_{0}\right)$. It then follows from (2) that

$$
\left\langle u_{1}^{*}-u_{0}^{*}, v\right\rangle \geqslant 0
$$

which contradicts (1). Therefore $T$ is $D$-maximal monotone.

The following theorem is a corollary of Theorems 1 and 2 and generalizes Theorem 1 of [4].

THEOREM 3. Let $X$ be a locally convex Hausdorff space, and $T$ a monotone operator from $X$ into $2^{X^{*}}$ with a quasi-dense domain $D(T)$. Suppose that $T$ is locally bounded at each point of $D(T)$, and $T(u)$ is a weak*-closed convex subset of $X^{*}$ for each $u \in D(T)$. Then $T$ is upper demicontinuous if and only if it is upper hemicontinuous.

Remark. If $X$ is a Fréchet space, then a monotone operator $T$ from $X$ into $2^{X^{*}}$ is locally bounded at each interior point of $D(T)$ (see [2]). A consequence of this result and of Theorem 3 is Theorem 4 which for single-valued operators $T$ is obtained in [3].

THEOREM 4. Let $X$ be a Fréchet space, and $T$ a monotone operator from $X$ into $2^{X^{*}}$ with $D(T)$ open in $X$. Suppose that for each $u \in D(T), T(u)$ is a weak ${ }^{*}$-closed and convex subset of $X^{*}$. Then $T$ is upper demicontinuous if and only if it is upper hemicontinuous.

ACKNOWLEDGEMENT. The author is grateful to the referee of an earlier version of this manuscript for some useful comments.

\section{REFERENCES}

1. F. Browder, Continuity properties of monotone nonlinear operators in Banach spaces, Bull. Amer. Math. Soc. 70 (1964), 551-553. MR 29 \#502.

2. P. Fitzpatrick, P. Hess and T. Kato, Local boundedness of monotone-type operators, Proc. Japan Acad. 48 (1972), 275-277.

3. H. Junghenn, Demicontinuity and hemicontinuity in Fréchet space, Proc. Amer. Math. Soc. 38 (1973), 89-91.

4. T. Kato, Demicontinuity, hemicontinuity and monotonicity, Bull. Amer. Math. Soc. 70 (1964), 548-550. MR 29 \#501.

Department of Mathematics, National Technical University of Athens, Patission 42, GREECE 